\title{
Gráficos de probabilidade normal para avaliação de mecanismos de distribuição de sementes em semeadoras
}

\author{
Normal probability plots for evaluation of seeds distribution \\ mechanisms in seeders
}

\author{
Daniel Albiero ${ }^{1 *}$; Antonio José da Silva Maciel2 $^{2}$ K Kleber Pereira Lanças³; \\ Leonardo de Almeida Monteiro ${ }^{1}$; Carlos Alberto Viliotti ${ }^{1}$; Renildo Luis Mion ${ }^{1}$
}

\begin{abstract}
Resumo
Atualmente grande ênfase é dada para mecanismos dosadores de sementes, que devem atender exigências cada vez maiores em relação à qualidade de distribuição longitudinal, principalmente em relação ao índice de falhas, quebras e duplas. A avaliação destas variáveis demanda muito tempo e trabalho, tanto para obtenção de dados como para processamento. O objetivo deste trabalho foi propor a utilização de gráficos de probabilidade normal para facilitar o tratamento dos dados relativos aos fatores que possivelmente influem na qualidade de distribuição e diminuir o tempo de processamento dos dados de distribuição das sementes em semeadoras. A metodologia de avaliação constitui-se na medida dos espaçamentos entre sementes e contagem de sementes quebradas, espaçamentos falhos e sementes duplas. Foram realizados experimentos por meio de combinações de tratamentos cujos fatores de variação foram: o nível de sementes no reservatório, o nivelamento transversal da semeadora, a velocidade de deslocamento e a dosagem de sementes. Uma avaliação de semeadora provida de anel interno rotativo foi realizada em duas partes primeiramente com experimentos para construção dos gráficos de probabilidade normal e posteriormente em experimentos com maior amostragem de sementes para avaliação da influência dos fatores mais importantes detectados pelos gráficos de probabilidade normal. Verificou-se que a quantidade de dados necessários para a avaliação da semeadora é menor por meio da utilização dos gráficos de probabilidade normal, pois estes possibilitam priorizar os fatores significativos. A dosagem de sementes foi o fator que mais interferiu nas avaliações propostas, pois o fator (D) obteve maior significância.
\end{abstract}

Palavras-chave: Semeadoras de precisão, métodos gráficos, distribuição de sementes

\begin{abstract}
Currently great emphasis is given for seed metering that assist rigorous demands in relation to longitudinal distribution of seeds, as well as to the index of fails in spacing laws, breaks and double seeds. The evaluation of these variable demands much time and work of attainment of data and processing. The objective of this work went propose to use of graphs of normal probability, facilitating the treatment of the data and decreasing the time of processing. The evaluation methodology consists in the counting of broken seeds, fail spacing and double seeds through the measure of the spacing among seeds, preliminary experiments through combinations of treatments had been carried through whose factors of variation were the level of the reservoir of seeds, the leveling of the seed metering, the speed of displacement and
\end{abstract}

\footnotetext{
${ }^{1}$ Profs. Drs. do Dept ${ }^{0}$ de Engenharia Agrícola, Centro de Ciências Agrárias, UFC, Fortaleza, CE. E-mail: daniel.albiero@gmail. com; aiveca@ufc.br; viliotti@ufc.br; rion@ufc.br

${ }^{2}$ Prof. Dr. do Dept ${ }^{\circ}$ de Máquinas Agrícolas, Faculdade de Engenharia Agrícola, UNICAMP, Campinas, SP. E-mail: amaciel@ feagri.unicamp.br

${ }^{3}$ Prof. Dr. do Dept ${ }^{\circ}$ de Engenharia Rural, Faculdade de Ciências Agronômicas, UNESP, Botucatu, SP. E-mail: kplancas@gmail.com

* Autor para correspondência
} 
dosage of seeds. The evaluation was carried through in two parts, first through preliminary experiments for elaboration of the graphs of normal probability and later in experiments with bigger sampling for evaluation of the influence of the factors most important. It was done the evaluation of seed metering of rotating internal ring, and the amount of necessary data for the evaluation was very decreased through of the graphs of normal probability that facilitated to prioritize only the significant factors. The dosage of seeds was factor that more important because factor (D) have greater significance.

Key words: Precision planter, graphic methods, probability

\section{Introdução}

Quando é necessário estudar efeitos de vários fatores sobre a resposta deve-se realizar experimentos com delineamentos fatoriais. A categoria de projetos fatoriais $2^{\mathrm{k}}$ é particularmente útil em estágios iniciais onde existem muitos fatores a serem investigados (MONTGOMERY, 1991). Porém, segundo Cargnelutti et al. (2007) a disponibilidade de tempo, de mão-de-obra, de dados e de recursos financeiros e humanos, dificulta a realização deste processo, necessitando estimar o parâmetro populacional por meio de medições realizadas em um determinado número de elementos da população (amostra). Neste contexto Machado et al. (2009) coloca que o principal obstáculo à utilização operacional desses modelos é o elevado custo para obtenção das informações acerca das áreas amostradas, fato confirmado por Adami et al. (2010).

Heo et al. (2008) e Smith et al. (2009) afirmam que o mais potente e fácil método para avaliar a influência de fatores distintos em um experimento é o gráfico de probabilidade normal. Principalmente ao se considerar a quantidade de tempo necessário para a coleta, descrição, análise e discussão dos mesmos (KOZAK, 2010).

Segundo Silva (2004) a observação de um dado fenômeno pode resultar em um gráfico cuja análise fornece indicações que possibilitam descrever qualitativa e quantitativamente tal fenômeno. Daniel (1976) descreveu um projeto fatorial $2^{4}$ usado para estudar a velocidade de avanço de uma semeadoraadubadora de fluxo contínuo em função de quatro fatores: carga de sementes, taxa de fluxo de sementes, velocidade rotacional, e tipo de mecanismo de semeadura. Foi utilizada a metodologia do gráfico de probabilidade normal dos efeitos estimados para identificar quais fatores e interações de fatores são significativas e concluiu que os gráficos de probabilidade normal são um método estatístico muito interessante para economizar tempo de processamento pela diminuição do número de fatores que necessitam ter normalidade.

Segundo Kurachi et al. (1989) a maioria dos métodos de avaliação de semeadoras (DEA, ISO, ABNT e INTA ADAPTADO) tem como avaliação de dispersão o coeficiente de variação (CV\%) que é baseado no desvio padrão (DP), mas o seu cálculo leva em conta apenas parte da população e estará sempre sujeito à crítica pela razão óbvia de não refletir a variação total da amostra analisada. Este fato é essencialmente importante quando o primeiro fator a ser destacado é a dosagem de sementes (CASÃO JUNIOR, 2006). Portanto preocupação com normalidade dos dados são essenciais (ALBIERO et al., 2012).

Segundo Carolla, Sanches e Montiel (2007), ao se confeccionar um modelo multifatorial permitese estimar a combinação inicial apropriada dos fatores pertinentes, embora isto demande muita mão de obra. No entanto, desde que adequadamente subsidiado por gráficos de probabilidade normal, o trabalho pode ser reduzido substancialmente (FRAGIADAKIS; KARLIS; MEINTANIS, 2009).

Com a utilização de gráficos de probabilidade normal pode ser possível definir quais fatores em relação aos parâmetros de qualidade de semeadura que são realmente significativos e importantes, isto possibilita concentrar esforços nestes fatores diminuindo tempo de obtenção e processamento dos dados tais como espaçamento entre sementes, número de sementes quebradas, etc. Mukhopadhyay 
e Silva (2008) afirmam que ao se utilizar um gráfico de probabilidade normal é possível fixar o tamanho da amostra dos parâmetros importantes, não exigindo assim amostragens com muitos dados. Hines et al. (2006) e Snedecor e Cochran (1989) afirmam que quanto maior as amostras em relação ao nível de variabilidade, menor serão os erros na avaliação da análise de variância, pois maior a chance de ser atendida a exigência de normalidade na distribuição, validando o desvio padrão como medida de dispersão.

Armas e Herrera (2008) mostram que sempre é possível dispor de diversos tamanhos de amostras em relação a uma coleção de dados, e que a principal limitação da amostragem é o limite de confiança requerido sendo importante selecionar os parâmetros em um experimento que realmente tenham influencia no mesmo, para evitar perdas de tempo.

Os gráficos de probabilidade normal são métodos baseados na diagnose de detecção da validade ou não de um modelo ou análise de dados (MUSHKUDIANI; EINMAHL, 2007). Segundo Barros Neto, Scarminio e Bruns (2003) a metodologia do gráfico de probabilidade normal se baseia na noção de probabilidade cumulativa que representa a probabilidade de que o valor observado para a variável aleatória seja no máximo igual ao valor definido pelo ponto, aumentando continuamente à medida que a variável se desloca, tendendo a 1 . A curva que representa é monotonicamente crescente em forma de $\mathrm{S}^{3}$, que vai de 0 a 1 , podendo ser transformada em uma reta por meio de transformação da escala do eixo das ordenadas. Se os pontos da distribuição estiverem fora da reta conclui-se que os dados não seguem uma distribuição normal e a suposição de população homogênea está errada.

Kozak (2009) afirma que a análise de variância (ANOVA) é forma clássica de análise estatística mais usada na agricultura, no entanto é frequentemente usada de forma inapropriada: uma vez que ela é usada sem serem feitas maiores considerações em relação a suas restrições, como exigência de normalidade. Neste contexto é interessante a utilização do gráfico de probabilidade normal tanto para análise da normalidade dos dados como para escolha de parâmetros mais importantes.

O objetivo deste trabalho foi propor a utilização de gráficos de probabilidade normal para facilitar o tratamento dos dados e diminuir o tempo de processamento dos dados de distribuição dos mecanismos de distribuição de sementes em semeadoras.

\section{Material e Métodos}

O trabalho foi desenvolvido no Campo Experimental da Faculdade de Engenharia Agrícola/UNICAMP. Na Figura 1 tem-se o desenho conceitual da unidade semeadora de anel interno rotativo (CHANG, 1998). A regulagem de dosagem é feita com a troca do anel de sementes.

Neste trabalho foi seguido o tratamento definido por Kurachi et al. (1989) denominado DEA que considera obrigatoriamente os seguintes fatores: nível de sementes no reservatório (cheio (1/1) e a um quarto (1/4); velocidade de operação da semeadora $\left(0,694 \mathrm{~m} \mathrm{~s}^{-1}\left(2,5 \mathrm{~km} \mathrm{~h}^{-1}\right) ; 1,11 \mathrm{~m} \mathrm{~s}^{-1}\left(4,0 \mathrm{~km} \mathrm{~h}^{-1}\right)\right.$, regulagens básicas do mecanismo (dosagem 15 sementes/mà $6,6 \mathrm{~cm}$ entre sementes) e (10 sementes/ mà $10 \mathrm{~cm}$ entre sementes) e posições do equipamento ensaiado em relação ao solo ((nivelado longitudinal e transversalmente) e (inclinado $11^{\circ}$ a direita e nivelado longitudinalmente).

Para efeito de tratamento de dados em relação a influência dos fatores, estes foram codificados da seguinte forma: Eabcd onde (E) significa Experimento, (a) é o nível do tanque, (b) é a velocidade de deslocamento, (c) é o nivelamento da semeadora e (d) é a dosagem de sementes $\mathrm{m}^{-1}$. O mecanismo dosador de anel interno rotativo foi montado no protótipo da semeadora multifuncional conservacionista (ALBIERO, 2006). 
Figura 1. Esquema da construção geral da unidade dosadora e seus componentes (CHANG, 1998), (a) vista geral; (b) detalhe do mecanismo ejetor.
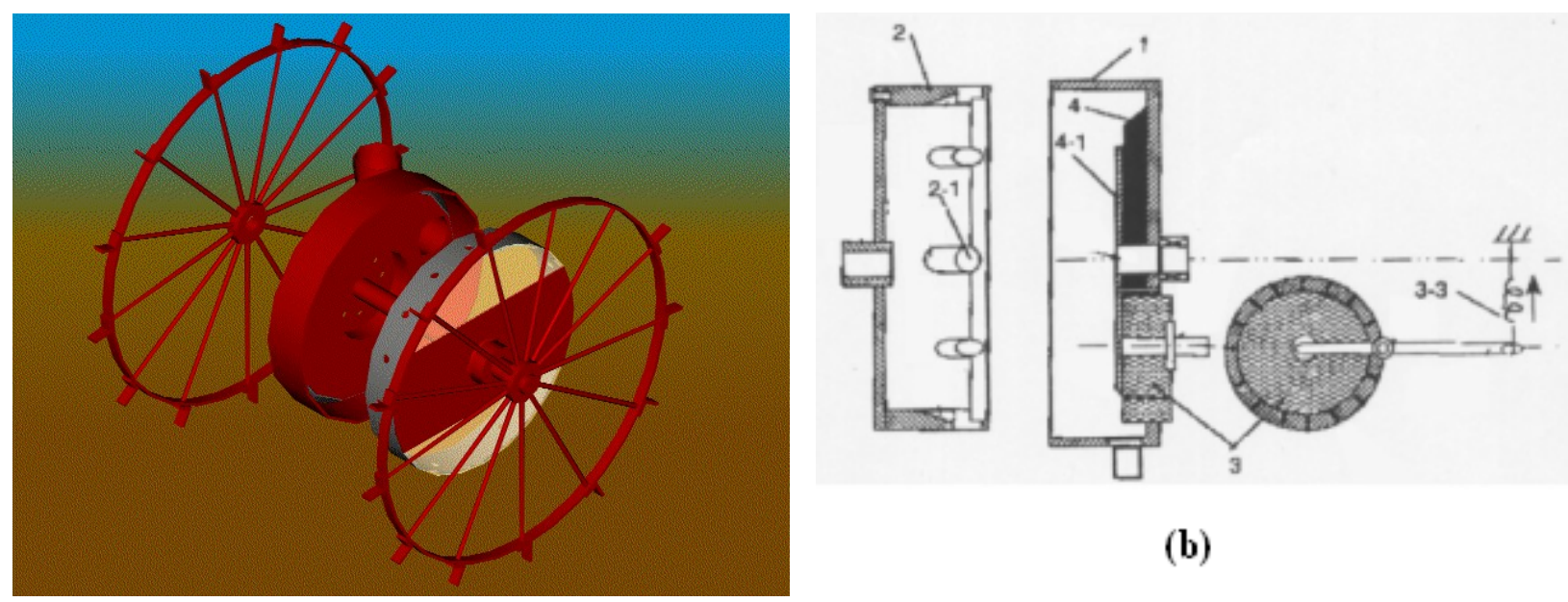

(b)

(a)

Fonte: Elaboração dos autores.

A máquina fez os tiros de semeadura sobre uma esteira formada por areia solta em pista de testes. Os elementos ativos de preparo do sulco e de cobrimento da semente foram retirados. Após a liberação das sementes pelo mecanismo as mesmas seguiam pelo tubo de condução de sementes e eram depositadas superficialmente sobre o leito de areia solta, que evitava que as sementes saltassem ou se distanciassem do local de deposição. A semeadura foi realizada com soja variedade BRS 232, com índice de germinação de $80 \%$ e pureza de $99 \%$, peneira $5,5 \mathrm{~mm}$. A profundidade de semeadura foi regulada para $5 \mathrm{~cm}$ e espaçamento entre linhas de $0,5 \mathrm{~m}$.

A metodologia utilizada para a avaliação de mecanismos de semeadura e adubação foi descrita por Kurachi et al. (1989) e constitui-se na avaliação de dois tiros de semeadura com aproximadamente 250 espaçamentos para cada combinação de fatores. A Tabela 1 apresenta o planejamento experimental da avaliação da semeadora.

Adistribuição longitudinal foi avaliada medindose o espaçamento entre as sementes depositadas na esteira de areia em função dos seguintes critérios: 1-Espaçamento duplo: espaço entre sementes menor que metade do espaçamento referência (Xref);
2-Espaçamento falho: espaço entre sementes 50\% maior do que o espaçamento referência (Xref); 3- Sementes quebradas: todas as sementes que possuíam qualquer dano sobre sua superfície (arranhões, embriões danificados, dicotiledônes separados, ou alargados, etc).

A avaliação das sementes quebradas foi realizada segundo metodologia descrita por Marcondes, Miglioranza e Fonseca (2010) em que foi obtida pela razão entre a massa das mesmas e a massa total da respectiva amostra em percentagem.

Neste trabalho foi seguida recomendação de Barros Neto, Scarminio e Bruns (2003) que determina que o efeito somente é significativo a 95\% de confiança se seu valor ultrapassar a seguinte relação: Efeito $>t_{4}{ }^{*}$ desvio padrão do efeito, sendo $t_{4}$ o valor da distribuição t de Student considerando o teste bilateral para quatro graus de liberdade $(2,776)$.

Para o processamento dos dados foram utilizados os softwares MINITAB 15 e STATGRAPH Plus 4.1. O nível de significância requerido foi considerado como 5\%. Foi realizada uma análise de variância pelo teste $\mathrm{F}$ nos tratamentos que apresentaram diferenças significativas. 
Tabela 1. Planejamento fatorial $2^{4}$, considerando dois níveis dos fatores nível reservatório, velocidade de deslocamento, posição da semeadora e dosagem de sementes.

\begin{tabular}{ccccc}
\hline Combinação* & $\begin{array}{c}\text { Nível } \\
\text { Reservatório }\end{array}$ & $\begin{array}{c}\text { Velocidade } \\
\text { Deslocamento } \\
\left(\mathrm{m} \mathrm{s}^{-1}\right)\end{array}$ & $\begin{array}{c}\text { Posição da } \\
\text { adubadora }\end{array}$ & $\begin{array}{c}\text { Dosagem } \\
\left(\text { sementes m }^{-1}\right)\end{array}$ \\
\hline E1111 & 1 & 0,69 & nivelado & 10 \\
E1112 & 1 & 0,69 & nivelado & 15 \\
E1121 & 1 & 0,69 & inclinado & 10 \\
E1122 & 1 & 0,69 & inclinado & 15 \\
E1211 & 1 & 1,11 & nivelado & 10 \\
E1212 & 1 & 1,11 & nivelado & 15 \\
E1221 & 1 & 1,11 & inclinado & 10 \\
E1222 & 1 & 1,11 & inclinado & 15 \\
E2111 & $1 / 4$ & 0,69 & nivelado & 10 \\
E2112 & $1 / 4$ & 0,69 & nivelado & 15 \\
E2121 & $1 / 4$ & 0,69 & inclinado & 10 \\
E2122 & $1 / 4$ & 0,69 & nivelinado & 15 \\
E2211 & $1 / 4$ & 1,11 & nivelado & 10 \\
E2212 & $1 / 4$ & 1,11 & inclinado & 15 \\
E2221 & $1 / 4$ & 1,11 & inclinado & 10 \\
E2222 & $1 / 4$ & 1,11 & 15 \\
\hline
\end{tabular}

*Codificação: Eabcd, onde (a) é o nível do tanque, (b) é a velocidade de deslocamento, (c) é o nivelamento da semeadora e (d) é a dosagem $\mathrm{m}^{-1}$.

Fonte: Elaboração dos autores.

\section{Resultados e Discussão}

A patinagem da roda motora foi em média de $3,09 \%$ o que resulta em diminuição da dosagem de sementes. Assim, a dosagem de sementes cai para 14,5 sementes $\mathrm{m}^{-1}$ à6,9 $\mathrm{cm}$ entre sementes para $\mathrm{o}$ caso de dosagem desejada de 15 sementes $\mathrm{m}^{-1}$; e 9,7 sementes $\mathrm{m}^{-1}$ à10,3 cm entre semente para o caso de dosagem desejada de 10 sementes $\mathrm{m}^{-1}$. Assim para dosagem de sementes de 14,5 sementes $\mathrm{m}^{-1}$, o espaçamento duplo foi considerado como distância entre sementes menor que $3,25 \mathrm{~cm}$ e falha como distância entre sementes maior que $9,75 \mathrm{~cm}$; Para dosagem de 10 sementes $\mathrm{m}^{-1}$ o espaçamento duplo foi considerado como distância entre sementes menor do que $5 \mathrm{~cm}$ e falha como espaçamento entre sementes maior que $15 \mathrm{~cm}$. Estes parâmetros de espaçamento são essenciais para se atender o stand inicial de plantas recomendado agronomicamente.

Em função dos dados obtidos nos experimentos preliminares foram gerados os gráficos de probabilidade normal (Figuras 2, 34 e 5), e a Tabela 2 de efeitos dos fatores. Em função da análise destes foi possível direcionar os testes para os fatores pertinentes, sendo realizada duas repetições com 1000 espaçamentos em cada ensaio considerando as combinações dos fatores constantes na Tabela 3. 
Figura 2. Gráfico de Probabilidade normal da distribuição longitudinal de sementes, significância de 0,05 . (A $\rightarrow$ nível do reservatório de sementes; $\mathrm{B} \rightarrow$ velocidade deslocamento; $\mathrm{C} \rightarrow$ nivelamento da semeadora e $\mathrm{D} \rightarrow$ dosagem de sementes).

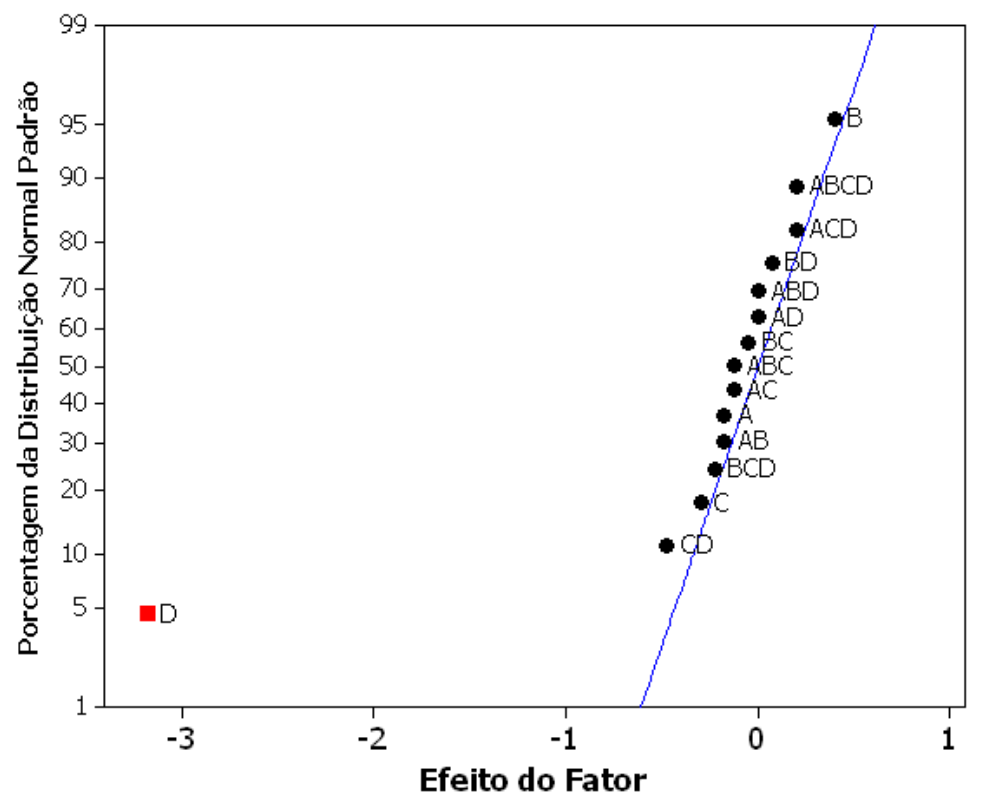

Fonte: Elaboração dos autores.

Figura 3. Gráficos de probabilidade normal de sementes duplas, significância de 0,05 . (A $\rightarrow$ nível do reservatório de sementes; $\mathrm{B} \rightarrow$ velocidade deslocamento; $\mathrm{C} \rightarrow$ nivelamento da semeadora e $\mathrm{D} \rightarrow$ dosagem de sementes).

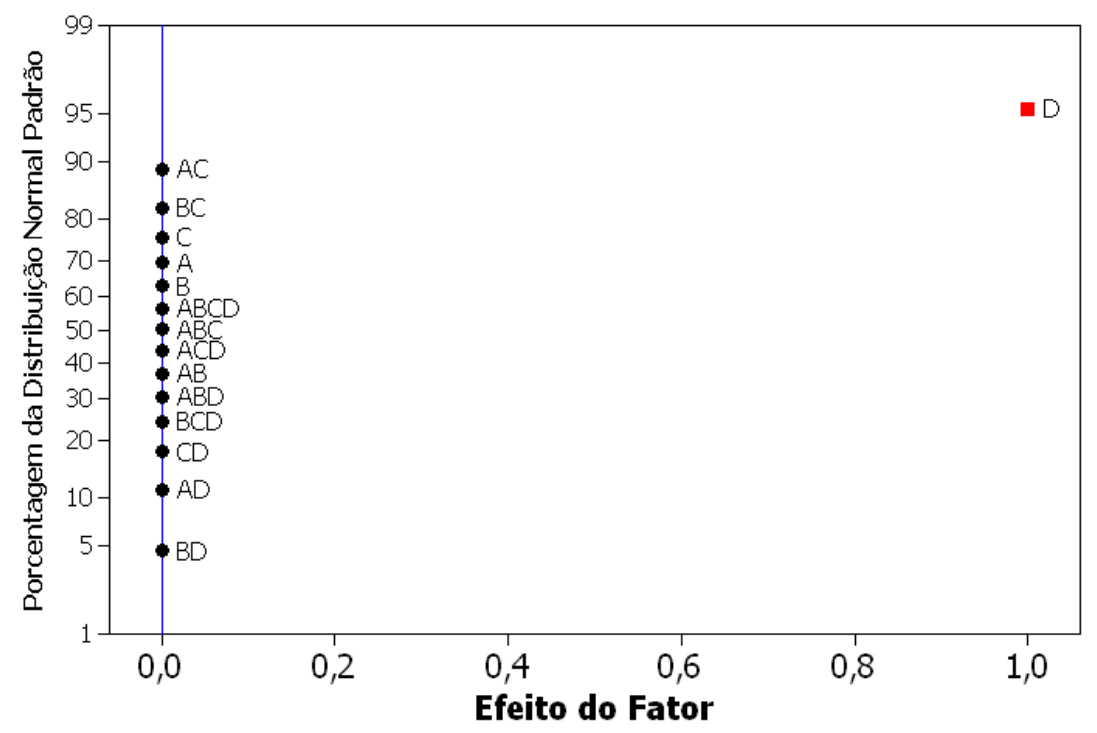

Fonte: Elaboração dos autores. 
Figura 4. Gráficos de probabilidade normal de sementes quebradas, significância de 0,05 . (A $\rightarrow$ nível do reservatório de sementes; $\mathrm{B} \rightarrow$ velocidade deslocamento; $\mathrm{C} \rightarrow$ nivelamento da semeadora e $\mathrm{D} \rightarrow$ dosagem de sementes).

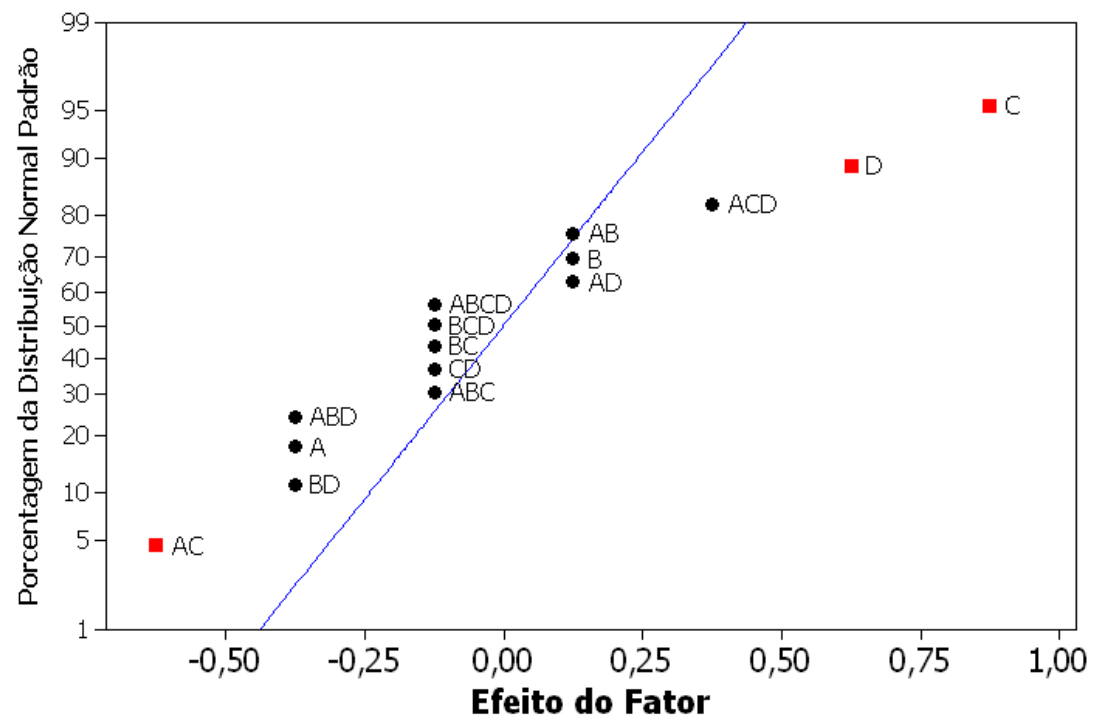

Fonte: Elaboração dos autores.

Figura 5. Gráficos de probabilidade normal de espaçamentos falhos, significância de 0,05 . (A $\rightarrow$ nível do reservatório de sementes; $\mathrm{B} \rightarrow$ velocidade deslocamento; $\mathrm{C} \rightarrow$ nivelamento da semeadora e $\mathrm{D} \rightarrow$ dosagem de sementes).

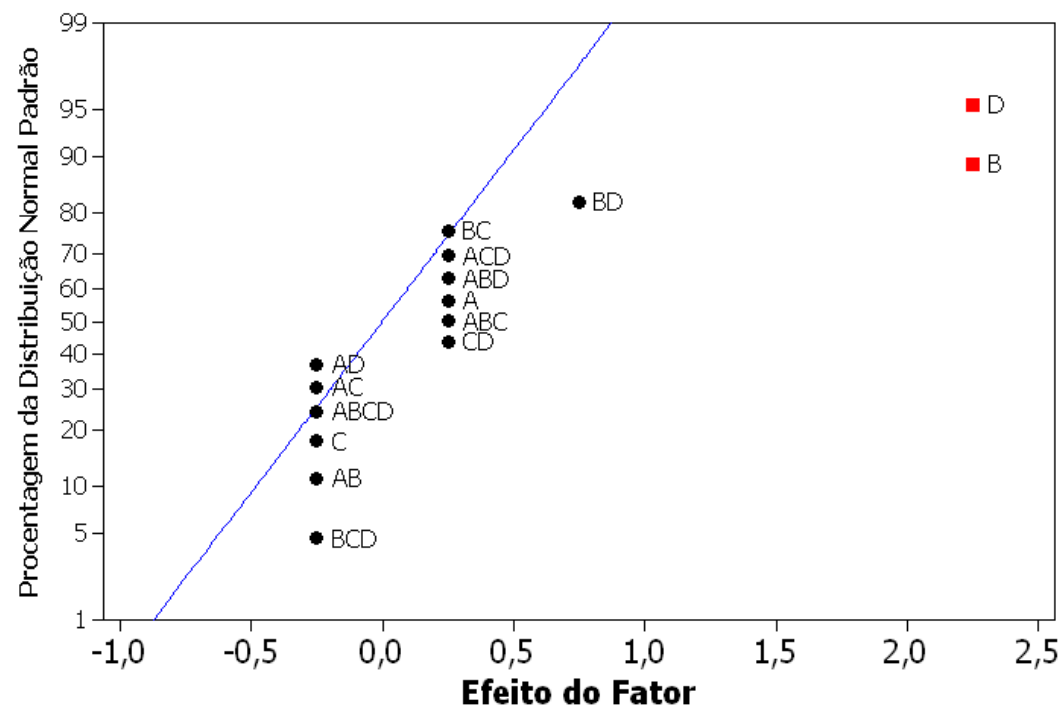

Fonte: Elaboração dos autores. 
Tabela 2. Análise de efeitos e desempenho da semeadora para a distribuição longitudinal, sementes quebradas, sementes duplas e falhas no espaçamento em função dos fatores (amostras de 250 sementes).

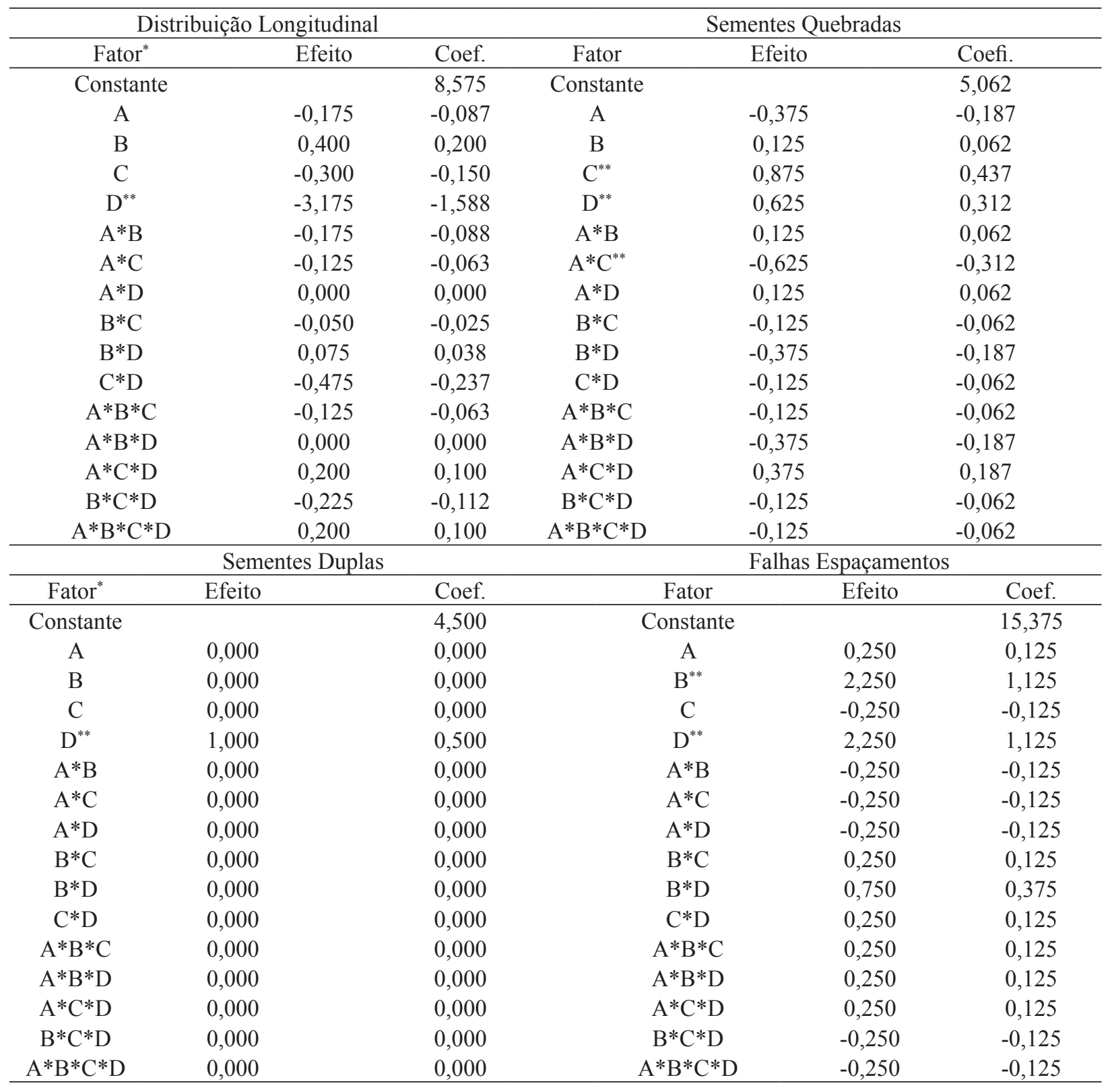

${ }^{*}$ Codificação: A, B, C, D, em que (A) é o nível do tanque, (B) é a velocidade de deslocamento, (C) é o nivelamento da semeadora e (D) é a dosagem $\mathrm{m}^{-1}$. ${ }^{* *}$ Fator Significativo a $95 \%$ do intervalo de confiança.

Fonte: Elaboração dos autores.

Pelos efeitos calculados para a distribuição longitudinal(Tabela 2), percebe-se que somente o fator dosagem de sementes (D), tem significado estatístico a $95 \%$ de confiança (Figura 2), indicando que todos os outros fatores não interferem na distribuição de sementes $\mathrm{m}^{-1}(\mathrm{C})$ o que indica a priorização da análise de variância a dois grupos homogêneos: distribuição longitudinal em função da dosagem D1 (10 sementes $\left.\mathrm{m}^{-1}\right)$ e D2 (15 sementes $\left.\mathrm{m}^{-1}\right)$.

Segundo Hines et al. (2006) o método do gráfico de probabilidade normal determina se os dados se adaptam a uma distribuição hipotética com base em um exame visual, se algum dos pontos não se comportar linearmente em função do conjunto dos 
dados, se este ponto tem significado estatístico, ou seja, não se comporta "normalmente". Cargnelutti et al. (2007) afirma que tal fato se deve essencialmente a um fator não muito importante na análise do fenômeno.

Assim na Figura 2, o ponto (D) merece melhor atenção devido ao grande deslocamento deste em relação à linha de normalidade além do comportamento muito diverso em relação ao conjunto de pontos relativos aos fatores estudados.

Esta fato indica um fator que influencia diretamente o resultado da semeadura, mostrando a forte influência da dosagem de sementes na variável distribuição longitudinal de sementes.

Já em relação a sementes quebradas pelo gráfico de probabilidade normal (Figura 4), percebe-se que existem três grupos bem distintos de influência dos fatores: Dosagem de sementes (D), posição da semeadora (C) e a interação entre dois fatores (AC).

O nível do reservatório e posição da semeadora têm significado físico claro, pois, a quebra de sementeséum fenômenorelacionadotantoà dosagem (mais ou menos sementes sendo distribuídas) como as questões geométricas e de posicionamento do sistema mecanismo/reservatório/sementes que define a orientação, direção e forma que a semente entra nos orifícios do anel.

Segundo Barros Neto, Scarminio e Bruns (2003) quanto mais afastados estiverem da região central da linha, seja pela direita ou esquerda, mais significativos são os efeitos dos fatores envolvidos, assim percebe-se que o fator (C) posição da semeadora é o que tem mais efeitos na quebra das sementes, seguido pelo fator (D) dosagem de sementes, o que é reiterado pelo gráfico apresentado, que possibilita uma abordagem precisa dos parâmetros pertinentes (KOZAK, 2010).

Os fatores de interação (AC) e (ACD) possuem grande influência no resultado da variável semente quebrada e estão ligados diretamente a condições de interrelacionamente entre os efeitos ocasionados pela posição da semeadora, a dosagem e o nível do reservatório.
A interação entre o nível do reservatório de sementes e a posição da semeadora tem relação com a combinação de dois fatores que tem influência nos fenômenos de quebra das sementes, pode-se considerar que o nível de sementes no reservatório influi na tensão que cada semente recebe ao se deslocar dentro do reservatório até o orifício de entrada da semeadora.

Enquanto que a posição da semeadora se refere à direção que a tensão da carga de semente se orienta, pois esta orientação determina a ação de forças de atrito sobre as sementes que se encontram na região inferior do reservatório. Ambos efeitos quando combinados potencializam ações físicas que geram danos sobre as sementes.

A Figura 3 demonstra a influência da dosagem de sementes (D) com a existência de sementes duplas, assim como a não influência de todos os outros efeitos o que indica que a saída de sementes duplas está intimamente ligada à velocidade de rotação do anel de semente. O resultado condiz com a afirmação de Heo et al. (2008) de que os gráficos de probabilidade normal são instrumentos sensíveis as influências ou não dos fatores de semeadura. Em relação aos espaçamentos falhos, a Figura 5 indica a influência direta da velocidade de deslocamento (B) com a dosagem de sementes (D), que estes fatores potencializam as falhas tanto devido a não entrada de sementes nos orifícios do anel como a velocidade de deslocamento linear da semeadora, o que gera maior espaçamento, portanto maior falha.

Em relação à qualidade de semeadura, por meio dos gráficos de probabilidade normal foram identificados os fatores mais influentes no processo e, assim, foram realizadas testes com maiores amostragens focadas nestes fatores (Tabela 2). Pela Tabela 3 tem-se valor máximo de 5,7\% de espaçamentos não aceitáveis da distribuição longitudinal (somando-se as porcentagens de sementes quebradas, duplas e espaçamentos falhos), o que representa regularidade de distribuição longitudinal de $94,35 \%$. 


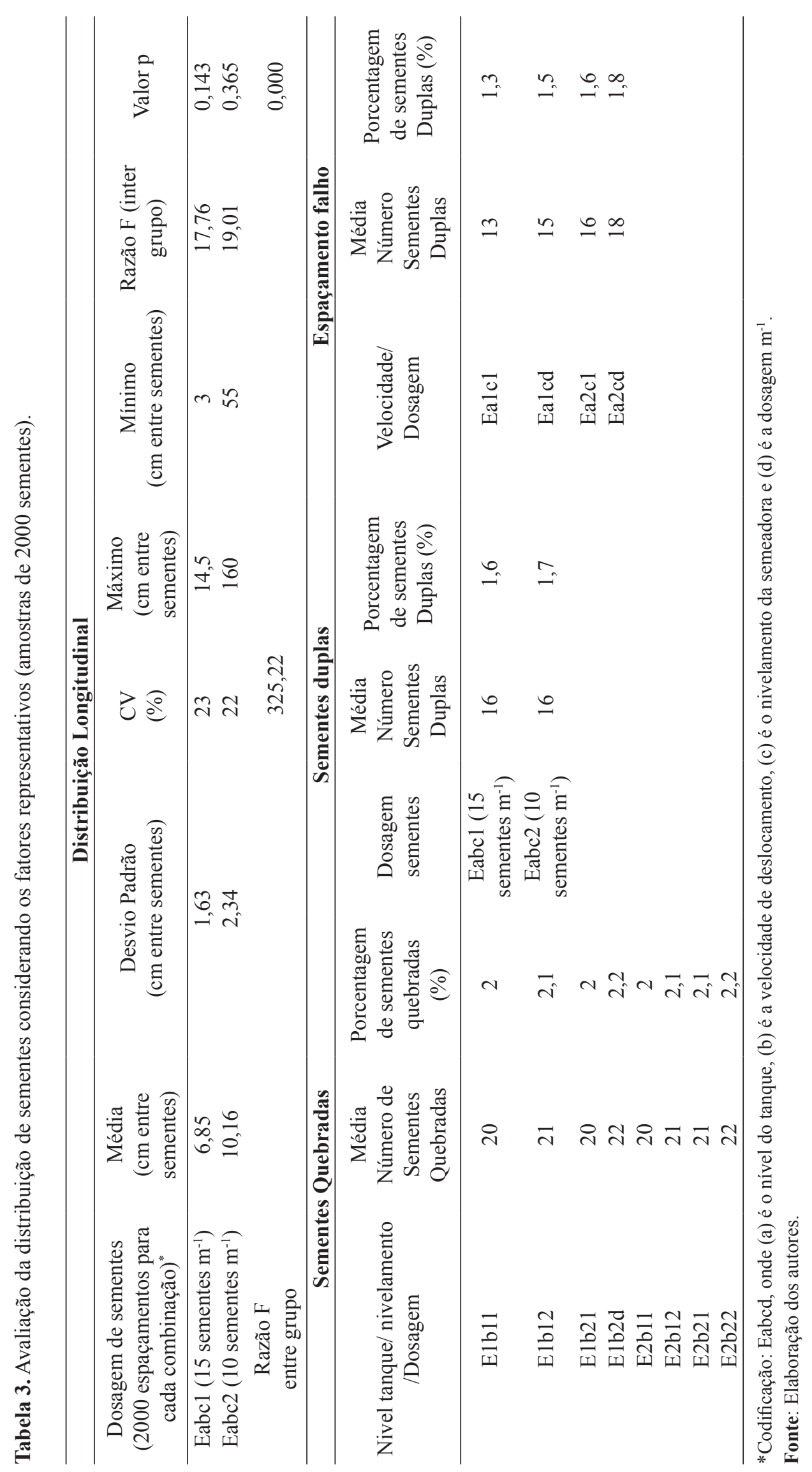




\section{Conclusões}

A metodologia estatística dos gráficos de probabilidade facilitou o tratamento dos dados relativos aos fatores que influem na qualidade de distribuição e diminuiu o tempo de processamento dos dados, pois possibilitou priorizar os fatores significativos. A dosagem de sementes foi o fator que mais interferiu nas avaliações propostas, pois o fator (D) obteve maior significância.

\section{Agradecimentos}

O primeiro autor agradece ao CNPq pela bolsa de estudos, a FINEP pelo apoio financeiro ao projeto e ao MDA do Governo Federal pelo apoio institucional. Os autores do trabalho agradecem a Cooperativa Agrícola da Região de Orlândia-SP (CAROL) pelo fornecimento das sementes.

\section{Referências}

ADAMI, M.; RIZZI, R.; MOREIRA, M. A.; RUDORFF, B. F. T.; FERREIRA, C. C. Amostragem probabilística estratificada por pontos para estimar a área cultivada de soja. Pesquisa Agropecuária Brasileira, Brasília, v. 45, n. 6, p. 585-592, 2010.

ALBIERO, D. Avaliação do preparo de solo empregando o sistema de cultivo conservacionista em faixas com "Paraplow" rotativo usando análise dimensional. 2006. Dissertação (Mestrado em Engenharia Agrícola) Universidade Estadual de Campinas, Campinas.

ALBIERO, D.; MACIEL, A. J. S.; MILAN, M.; MONTEIRO, L. A.; MION, R. L. Avaliação da distribuição de sementes por uma semeadora de anel interno rotativo utilizando média móvel exponencial. Revista Ciência Agronômica, Fortaleza, v. 43, n. 1, p. 8695, 2012.

ARMAS, S.; HERRERA, L. Amplitud de intervalos de confianza obtenidos por bonferroni de acuerdo al número de vaiables y del tamaño de la muestra en poblaciones multinormales. Revista de la Facultad de Ciencias Veterinarias, Caracas, v. 49, n. 2, p. 95-105, 2008.

BARROS NETO, B.; SCARMINIO, I. S.; BRUNS, R. E. Como fazer experimentos. Campinas: UNICAMP, 2003. $403 \mathrm{p}$.
CARGNELUTTI FILHO, A.; MATZENAUER, R.; MALUF, J. R. T.; SILVA, B. P. Tamanho de amostra para estimativa das médias decendiais de radiação solar global no Estado do Rio Grande do Sul. Ciência e Agrotecnologia, Lavras, v. 31, n. 5, p. 1402-1410, 2007.

CAROLLA, C.; SANCHES, R.; MONTIEL, E. Modelo estadistico que permite inferir concentración de potasio em composto producido a partir de desechos orgánicos. Revista de la Facultad de Ingenieria Universidad Central de Venezuela, Caracas, v. 22, n. 2, p. 125-132, 2007.

CASÃO JUNIOR, R. O. Máquinas e qualidade de semeadura em plantio direto. Revista Plantio Direto, Passo Fundo, v. 95, n. 5, p. 14-24, 2006.

CHANG, C. S. Patente da UNICAMP INPI (INPI 9802269-5). Semeadora de precisão com anel rotativo interno vertical e o distribuidor de adubo espiral cônica. Campinas: UNICAMP, 1998.

DANIEL, C. Applications of statistics to industrial experimentation. New York: John Wiley and Sons, 1976. $512 \mathrm{p}$.

FRAGIADAKIS, K.; KARLIS, D.; MEINTANIS, S. G. Tests of fit for normal inverse gaussian distributions. Statistical Methodology, Amsterdan, v. 6, n. 7, p. 559564, 2009.

HEO, J. H.; KHO, Y. W.; SHIN, H.; KIM, S.; KIM, T. Regression equations of probability plot correlation coeficient test stadistics from several probability distributions. Journal of Hydrology, Silsoe, v. 355, n. 2, p. 1-15, 2008.

HINES, W. W.; MONTGOMERY, D. C.; GOLDSMAN, D. M.; BORROR, C. M. Probabilidade e estatística na engenharia. Rio de Janeiro: LTC, 2006. 642 p.

KOZAK, M. Analyzing one-way experiments: a piece of cake or a pain in the neck? Scientia Agricola, Piracicaba, v. 66, n. 4 , p. $566-562,2009$.

Basic principles of graphing data. Scientia Agricola, Piracicaba, v. 67, n. 4, p. 483-494, 2010.

KURACHI, S. A. H.; COSTA, J. A. S.; BERNARDI, J. A.; COELHO, J. L. D.; SILVEIRA, G. M. Avaliação tecnológica de semeadoras e ou adubadoras: tratamento de dados de ensaios e regularidade de distribuição longitudinal de sementes. Bragantia, Campinas, v. 48, n. 2, p. 249-252, 1989.

MACHADO, J. C.; SOUZA, J. C.; RAMALHO, M. A. P.; LIMA, J. L. Stability of combining ability effects in maize hybrids. Scientia Agricola, Piracicaba, v. 66, n. 4, p. 494-498, 2009. 
MARCONDES, M. C.; MIGLIORANZA, E.; FONSECA, I. C. B. Qualidade de sementes de soja em função do horário de colheita e do sistema de trilha de fluxo radial e axial. Engenharia Agrícola, Jaboticabal, v. 30, n. 2, p. 315-321, 2010.

MONTGOMERY, D. C. Design and analysis of experiments. 3. ed. New York: John Wiley and Sons, $1991.610 \mathrm{p}$.

MUKHOPADHYAY, N.; SILVA, B. M. Theory and applications of a new methodology for the random sequential probability ratio test. Statistical Methodology, Amsterdan, v. 5, n. 8, p. 424-453, 2008.

MUSHKUDIANI, N.; EINMAHL, J. H. J. Generalized probability-probability plots. Journal of Statistical Planning And Inference, Silsoe, v. 137, n. 5, p. 738-752, 2007.
SILVA, W. P.; SILVA, C. M. P. S.; CAVALCANTE, C. G. B.; SILVA, D. D. P. S.; SOARES, I. B.; OLIVEIRA, J. A. S.; SILVA, C. D. P. S. "LAB Fit" ajuste de curvas. Revista Brasileira de Ensino de Física, Porto Alegre, v. 26, n. 4, p. 419-422, 2004.

SMITH, J. K.; SARMIENTO, L.; ACEVEDO, D.; RODRIGUEZ, M.; ROMERO, R. Un método participativo para mapeo de fincas y recoleccion de información agrícola aplicable a diferentes escalas espaciales. Interciencia, Caracas, v. 37, n. 7, p. 258-278, 2009.

SNEDECOR, G. W.; COCHRAN, W. G. Statistical methods. 8. ed. Ames: Iowa university Press, 1989. 550 p. 